\title{
Regionalized Life Cycle Greenhouse Gas Emissions of \\ Forest Biomass Use for Electricity Generation in the
}

\section{United States}

Hui Xu, ${ }^{1}$ Gregory Latta, ${ }^{2}$ Uisung Lee, ${ }^{1}$ Jan Lewandrowski, ${ }^{3}$ Michael Wang ${ }^{l}$

${ }^{1}$ Systems Assessment Group, Energy Systems Division, Argonne National Laboratory, 9700 South

Cass Avenue, Lemont, IL 60439, United States

${ }^{2}$ Department of Natural Resources and Society, University of Idaho, 875 Perimeter Drive MS 1139,

Moscow, ID 83844, United States

${ }^{3}$ Office of the Chief Economist, United States Department of Agriculture, 1280 Maryland Ave SW,

Washington, DC 20250, United States 


\section{Supporting Information Contents}

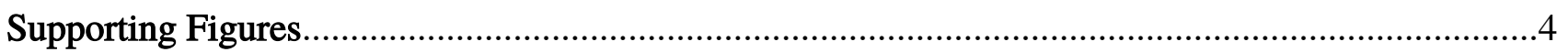

Figure S1. Locations of simulated bioelectricity facilities ..........................................................

Figure S2. System boundaries for pulp log, logging residue, lumber mill residue, and pellet-based pathways. Energy use and emissions associated with lumber mill operations are allocated between main product (lumber) and mill residues, using economic and mass allocation methods.................5

Figure S3. State-level life cycle GHG emissions of forest-residues-to-bioelectricity pathways .......6

( $\mathrm{g} \mathrm{CO}_{2} \mathrm{e} / \mathrm{kWh}$ ). Lumber mill share is based on mass-balance allocation.........................................6

Figure S4. State-level life-cycle GHG emissions $\left(\mathrm{g} \mathrm{CO}_{2} \mathrm{e} / \mathrm{kWh}\right)$ of forest-residues-to-bioelectricity pathways considering black carbon emissions. Lumber mill share is based on market-value

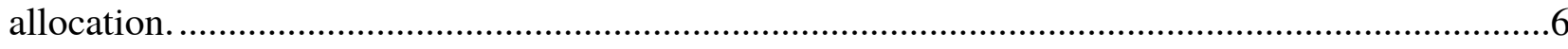

Figure S5. Boundaries of North American Electric Reliability Corporation (NERC) regions in the conterminous United States and spatial pattern of carbon intensities of grid electricity ( $\mathrm{g}$ $\mathrm{CO}_{2} \mathrm{e} / \mathrm{kWh}$ ) across NERC regions. Carbon intensities of electricity generation at the NERC level are obtained from the GREET model.

Figure S6. Sensitivity analysis results for the state-level life-cycle GHG emissions of forest to

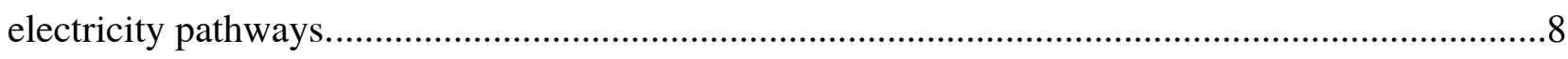

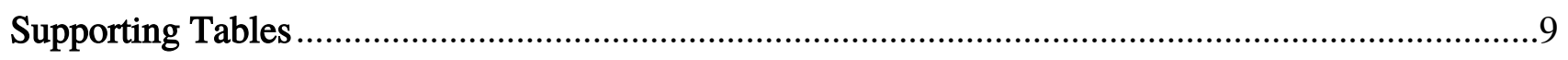

Table S1. Assumptions for the hypothetical woody biomass power plant. .....................................9

Table S2. Fuel and material inputs for site preparation, planting, and stand management. Natural regeneration is assumed for Northeast/North Central, thus there are no energy/material inputs. .....9 Table S3. Energy inputs for logging in the Pacific Northwest region. Data is adapted from Oneil et al. (2017). ${ }^{1}$ 10

Table S4. Energy inputs for logging in the Inland Pacific Northwest region. Data is adapted from Oneil et al. (2010). ${ }^{2}$ 
Table S5. Energy inputs for logging in the Northcentral region (except Tennesse). Data is adapted from Oneil et al. (2010). ${ }^{2}$

Table S6. Energy inputs for logging in Tennesse. Data is adapted from Abbas et al. (2018). ${ }^{3}$.......10

Table S7. Energy inputs for logging in the Northeast. Data is adapted from Quinn et al. (2020). ${ }^{4} 11$ Table S8. Energy inputs for logging in the Southeast region. Data is adapted from Lan et al. (in review)..$^{5}$

Table S9. Energy inputs for logging residues recovery. Data is adapted from Han et al. (2018) and Lan et al. (in review). ${ }^{5}$

Table S10. Energy inputs for wood pellets production. Data for Southeast and Pacific Northwest regions are derived from Morrison and Gordon $(2017)^{7}$ and Brackely et al. (2017), ${ }^{8}$ respectively.

Table S11. Break-down of life cycle GHG emissions by state for the pulpwood (softwood) to electricity pathway. States with zero transportation emissions indicate that pulpwood is not included in biomass supply mix simulated by LURA; values for other stages are included here for reference.

Table S12. Break-down of life cycle GHG emissions by state for the logging residues (softwood) to electricity pathway. States with zero transportation emissions indicate that logging residues are not included in biomass supply mix simulated by LURA; values for other stages are included here for reference.

Table S13. Break-down of life cycle GHG emissions by state for the mill residues (sawdust and shavings, softwood) to electricity pathway. States with zero transportation emissions indicate that mill residues are not included in biomass supply mix simulated by LURA; values for other stages are included here for reference.

Table S14. Break-down of life cycle GHG emissions by state for the wood pellet (sourced from pulpwood, softwood) to electricity pathway. States with zero transportation emissions indicate that mill residues are not included in biomass supply mix simulated by LURA; values for other stages are included here for reference 
Table S15. Break-down of life cycle GHG emissions by state for the wood pellet (sourced from pulpwood) to electricity pathway. States with zero transportation emissions indicate that mill residues are not included in biomass supply mix simulated by LURA; values for other stages are

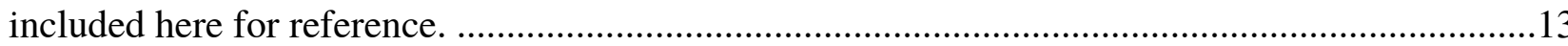

Table S16. Comparison of economic value versus mass based allocation factors used for the sawing process (log to rough green lumber) at lumber mill. States within the same region share the

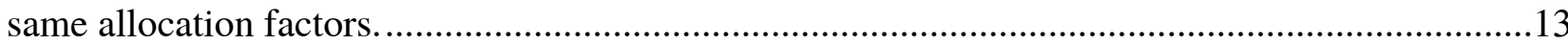

Table S17. Comparison of economic value versus mass based allocation factors used for the planning process (rough saw lumber to planned lumber) at lumber mill. States within the same region share the same allocation factors. 


\section{Supporting Figures}

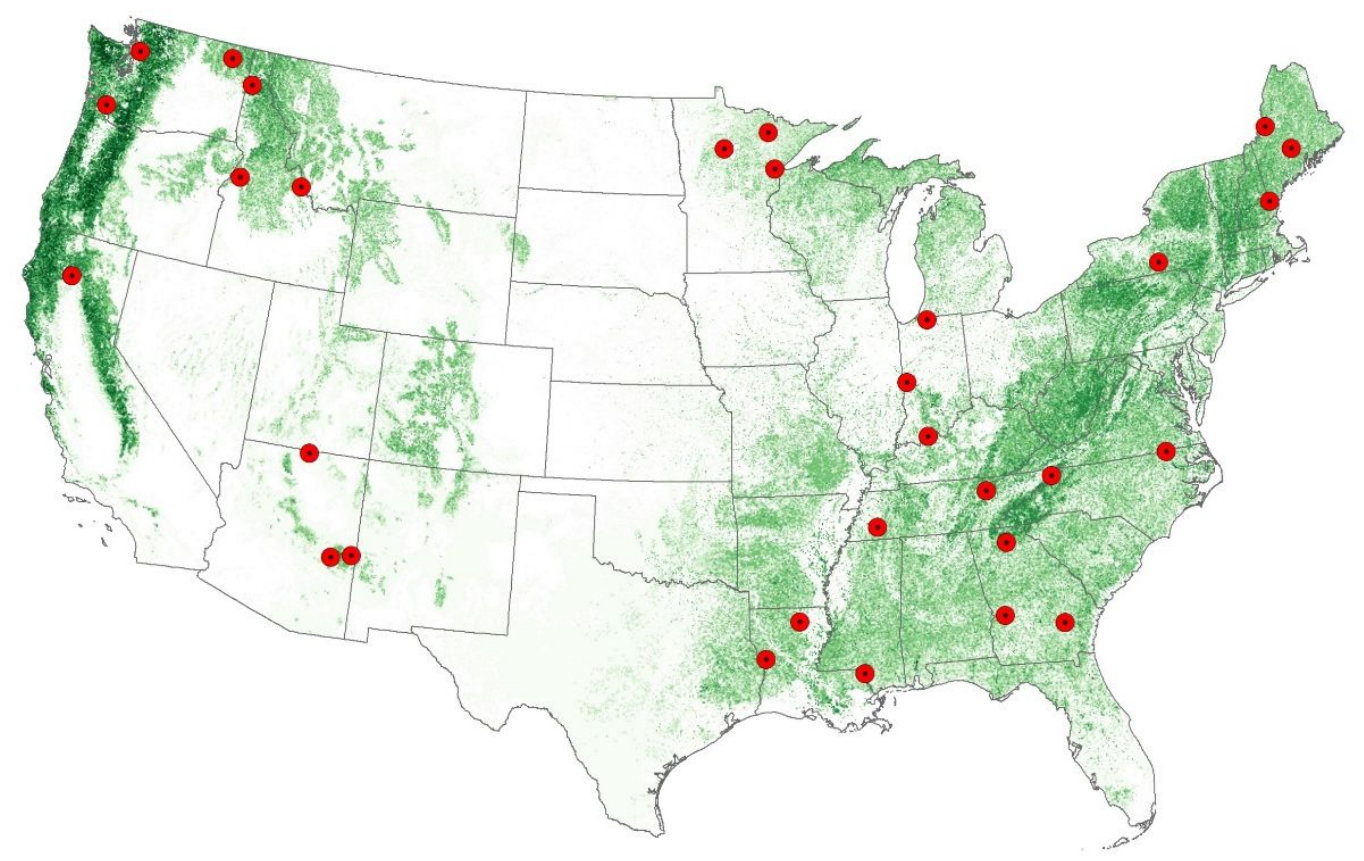

\section{Legend}

- Location of bio-power facilities State boundary

Above ground forest biomass (lb/acre)

- $>240,000$

- $120,000-240,000$

$60,000-120,000$

$30,000-60,000$

$<30,000$

Figure S1. Locations of simulated bioelectricity facilities 


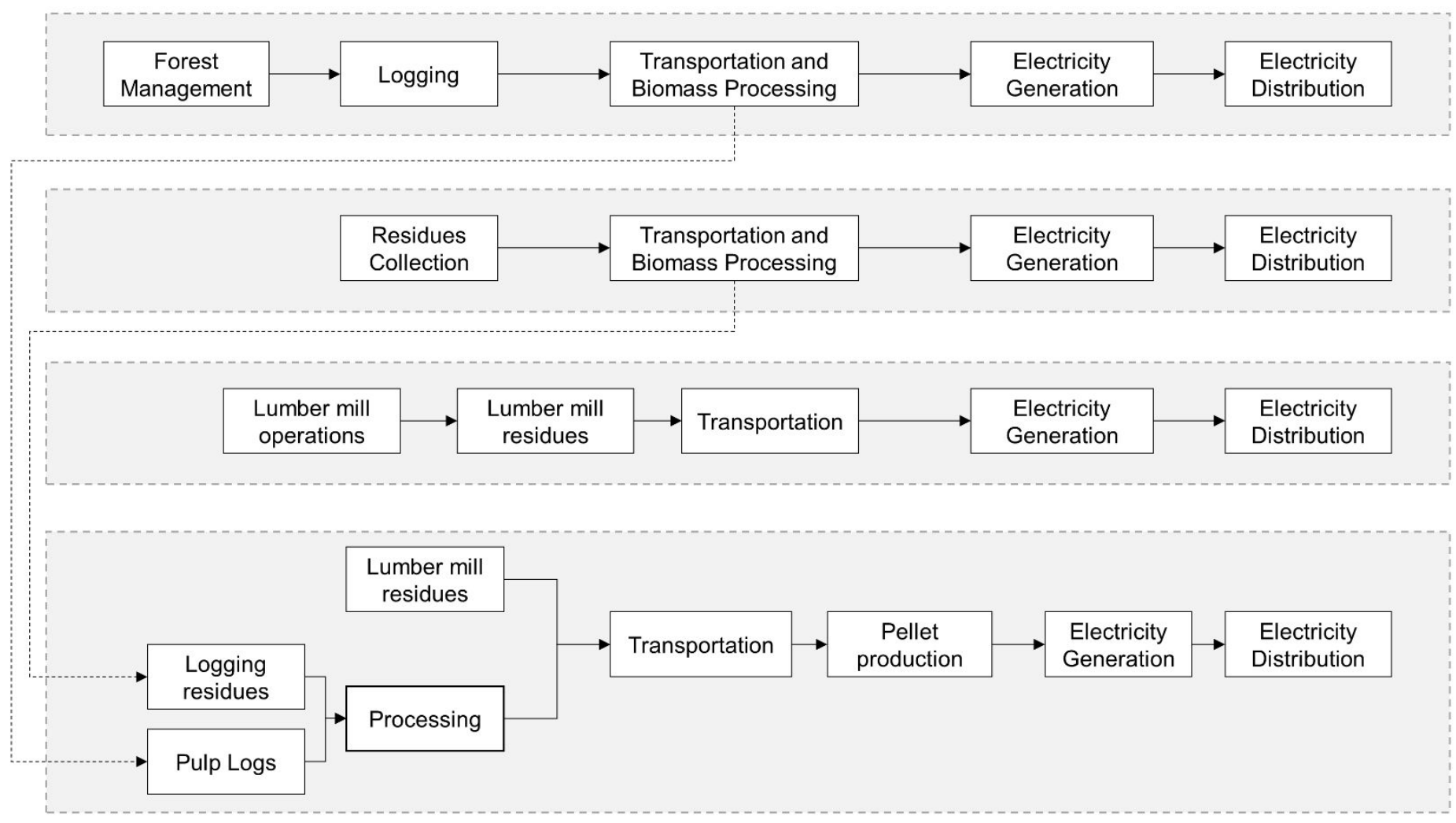

Figure S2. System boundaries for pulp log, logging residue, lumber mill residue, and pellet-based pathways. Energy use and emissions associated with lumber mill operations are allocated between main product (lumber) and mill residues, using economic and mass allocation methods. 


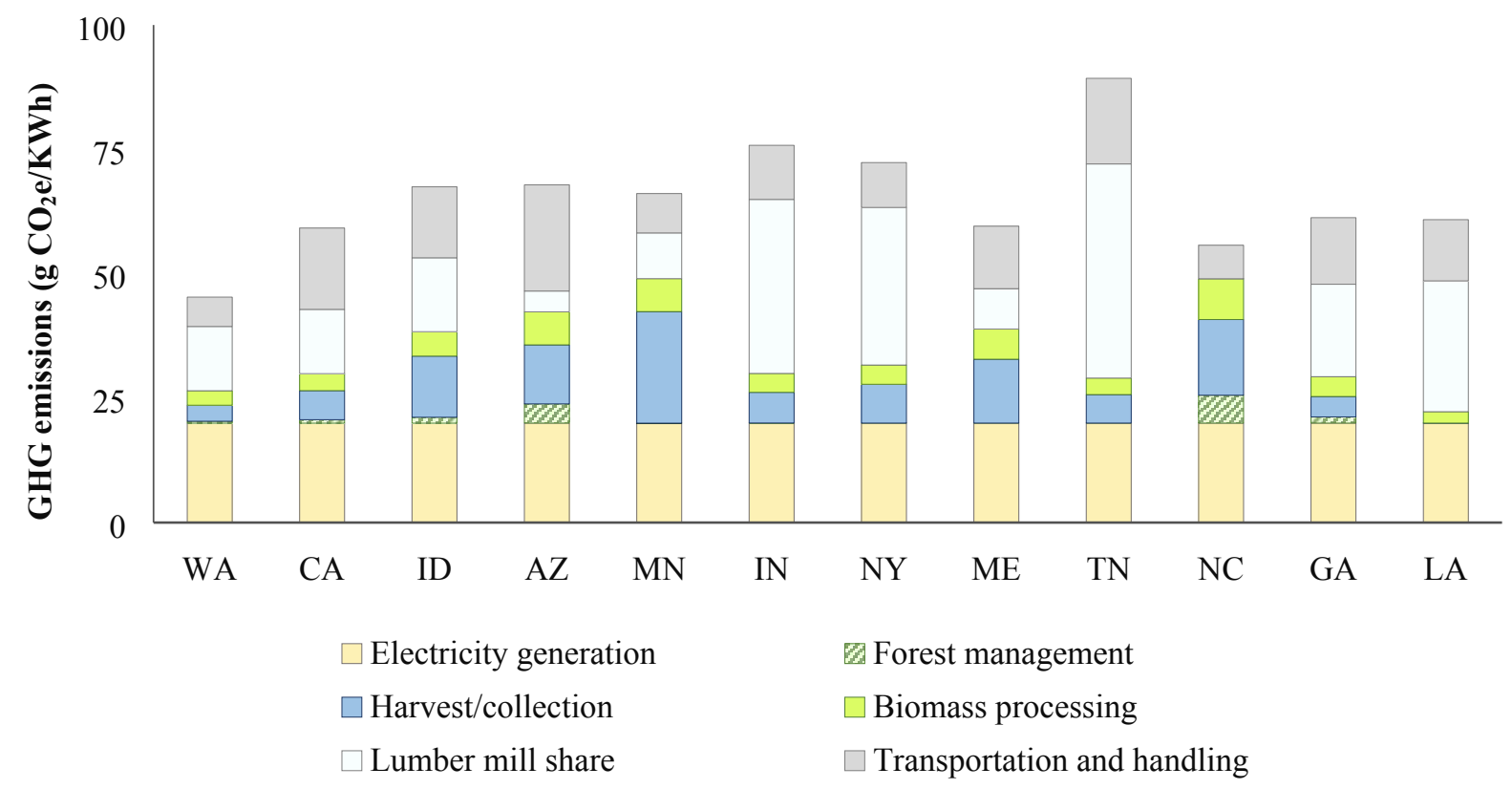

Figure S3. State-level life cycle GHG emissions of forest-residues-to-bioelectricity pathways $\left(\mathrm{g} \mathrm{CO}_{2} \mathrm{e} / \mathrm{kWh}\right)$. Lumber mill share is based on mass-balance allocation.

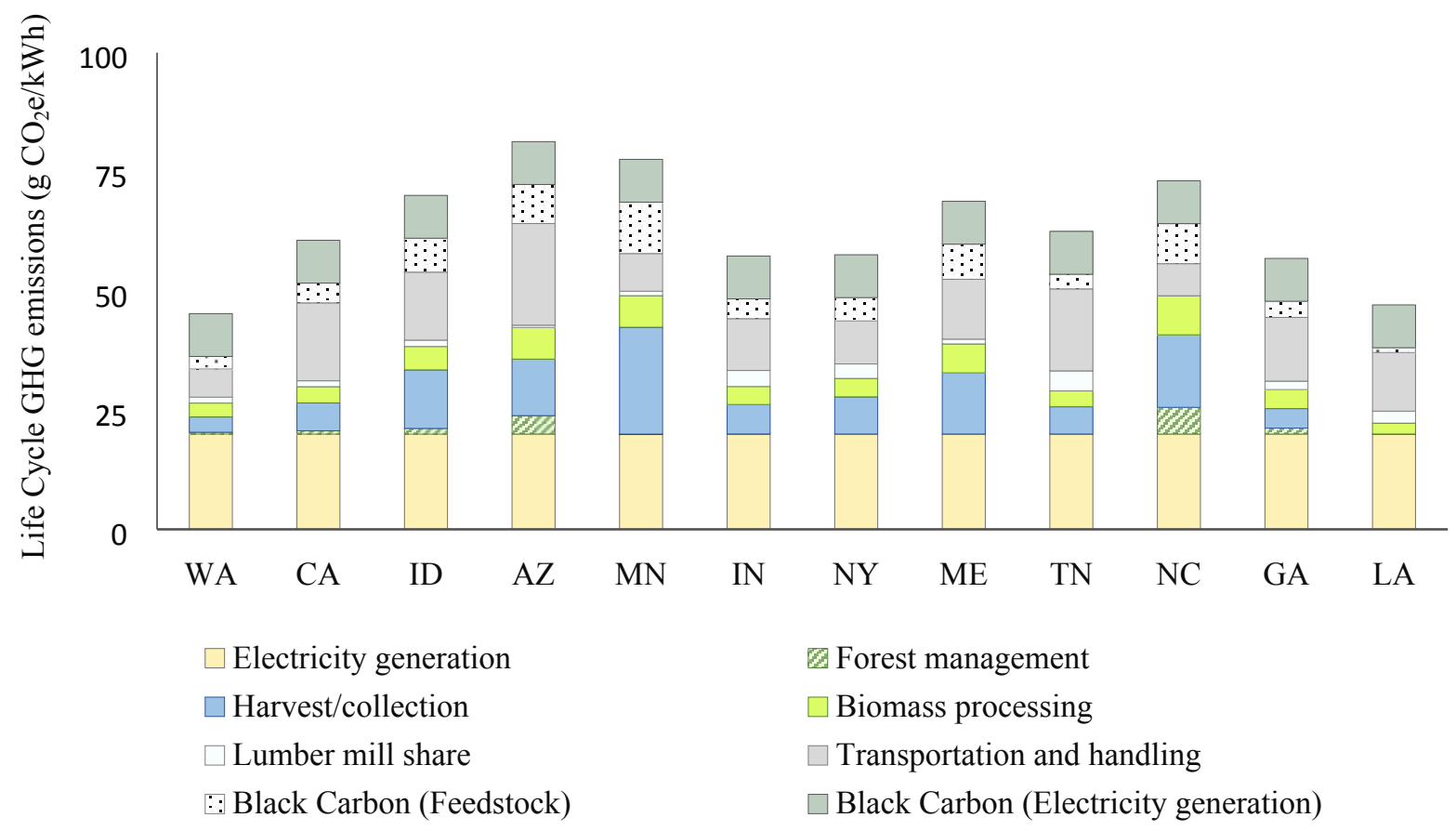

Figure S4. State-level life-cycle $\mathrm{GHG}$ emissions $\left(\mathrm{g} \mathrm{CO}_{2} \mathrm{e} / \mathrm{kWh}\right.$ )of forest-residues-to-bioelectricity pathways considering black carbon emissions. Lumber mill share is based on market-value allocation. 


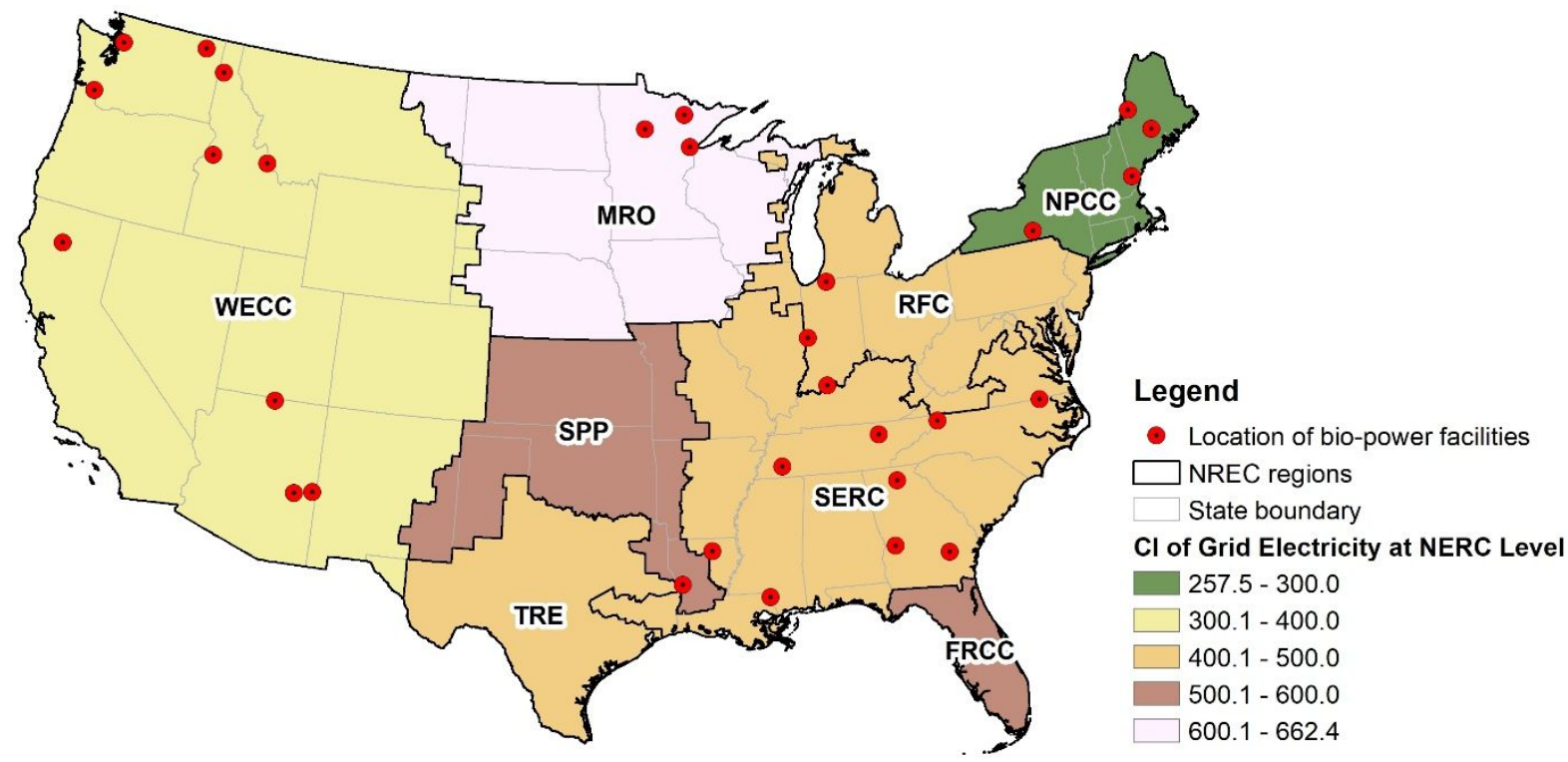

Figure S5. Boundaries of North American Electric Reliability Corporation (NERC) regions in the conterminous United States and spatial pattern of carbon intensities of grid electricity $\left(\mathrm{g} \mathrm{CO}_{2} \mathrm{e} / \mathrm{kWh}\right)$ across NERC regions. Carbon intensities of electricity generation at the NERC level are obtained from the GREET model. 
Figure S6. Sensitivity analysis results for the state-level life-cycle GHG emissions of forest to electricity pathways.

$$
\text { .100\% } 50.6 \%
$$

Transportation distance

Logging fuel input

Biomass processing

Lumber mill fuel input

Price of sawdust/shavings

Price of clean wood chips

Residues colleciton

Forest fertilizer use

Forest management fuel use

$$
-25 \%-25 \%
$$

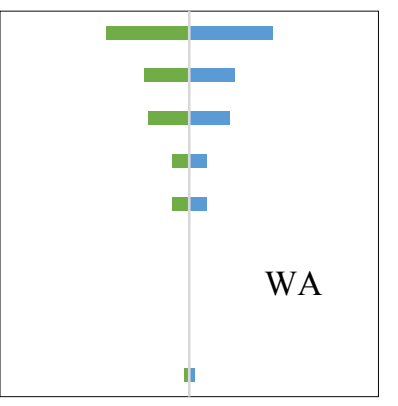

Changes in GHG emissions (\%)

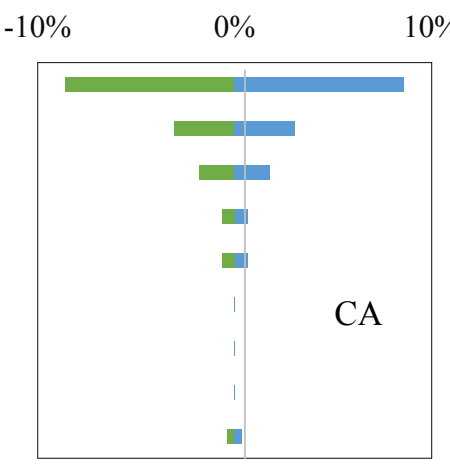

Changes in GHG emissions (\%)

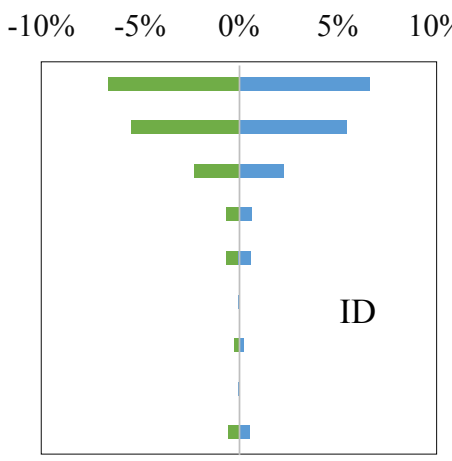

Changes in GHG emissions (\%)

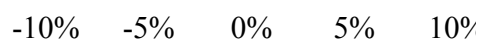

Changes in GHG emissions (\%)
$-10 \% \quad-5 \% \quad 0 \% \quad 5 \% \quad 10^{\circ}$

Transportation distance

Logging fuel input

Biomass processing

Lumber mill fuel input Price of sawdust/shavings

Price of clean wood chips

Residues colleciton

Forest fertilizer use

Forest management fuel use

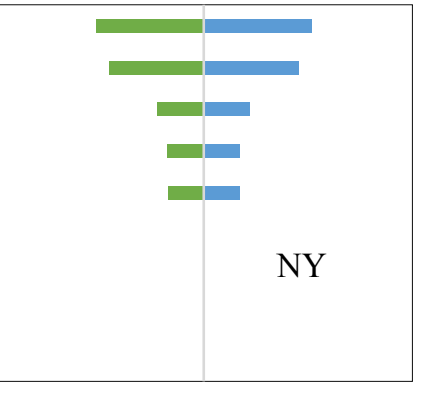

Changes in GHG emissions (\%)

$$
-10 \%
$$

$0 \%$

$10 \%$

Transportation distance

Logging fuel input

Biomass processing

Lumber mill fuel input

Price of sawdust/shavings

Price of clean wood chips

Residues colleciton

Forest fertilizer use

Forest management fuel use

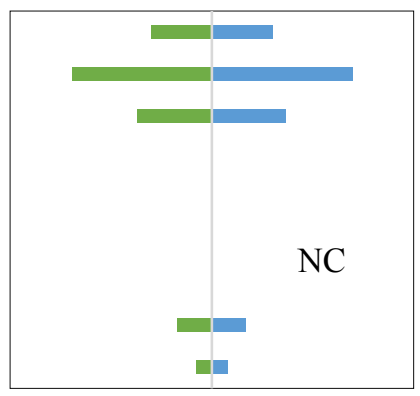

Changes in GHG emissions (\%)

$$
-10 \%
$$

$0 \%$

$10 \%$

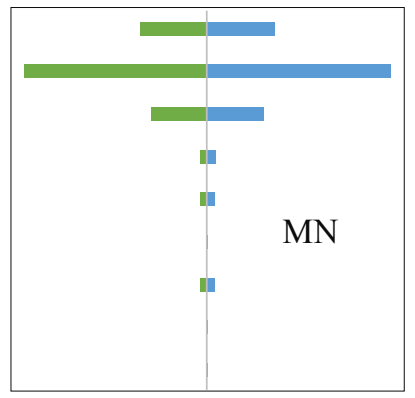

Changes in GHG emissions (\%)
$0 \%$

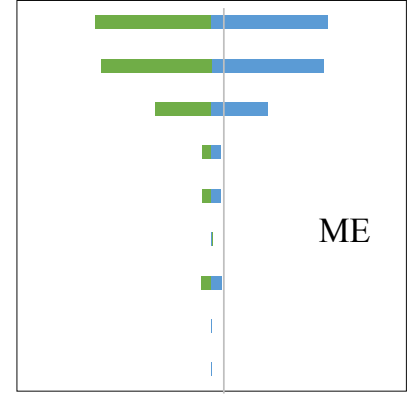

Changes in GHG emissions (\%)
$-10 \% \quad-5 \% \quad 0 \% \quad 5 \% \quad 10 \%$

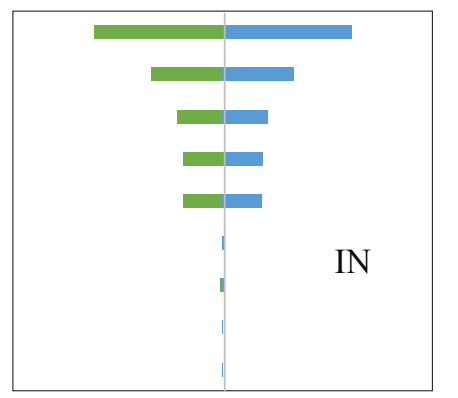

Changes in GHG emissions (\%)
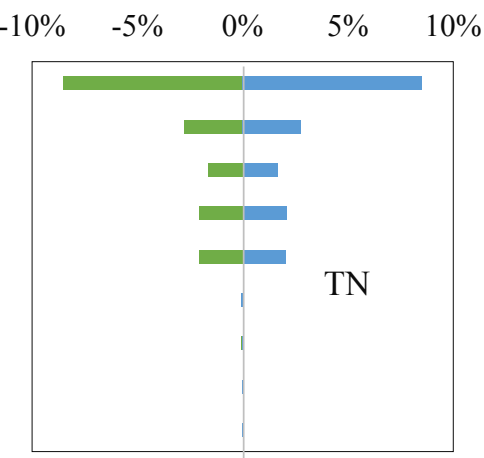

Changes in GHG emissions (\%)

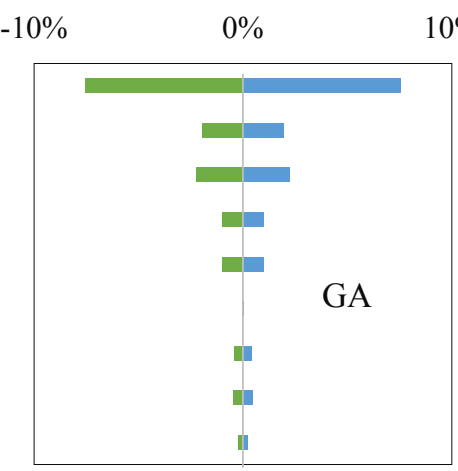

Changes in GHG emissions (\%)
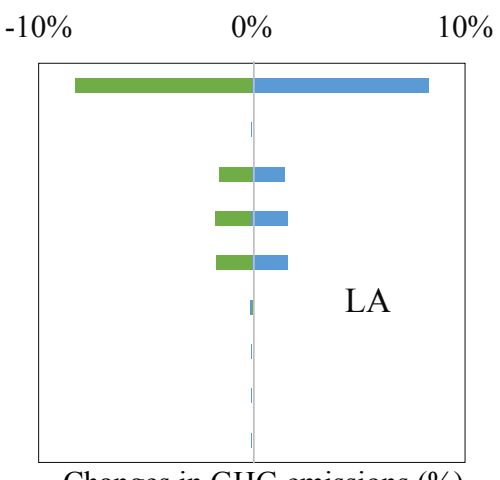

Changes in GHG emissions (\%) 


\section{Supporting Tables}

Table S1. Assumptions for the hypothetical woody biomass power plant.

\begin{tabular}{lrl}
\hline \multicolumn{1}{c}{ Variable } & \multicolumn{1}{c}{ Value } & \multicolumn{1}{c}{ Unit } \\
\hline Nameplate Capacity & 20 & $\mathrm{MW} / \mathrm{yr}$ \\
Heat Rate & 15.826 & $\mathrm{MJ} / \mathrm{kWh}$ \\
$\mathrm{HHV}$ & 13.956 & $\mathrm{MJ} / \mathrm{kg}$ biomass (dry) \\
Capacity Factor & 0.6 & Fraction of production time \\
Annual Generation & 105,120 & $\mathrm{MWh} / \mathrm{yr}$ \\
$\begin{array}{l}\text { Feedstock Required } \\
\text { Moisture content of } \\
\text { pulpwood feedstock }\end{array}$ & 119,204 & $\mathrm{t} / \mathrm{yr}$ (dry basis) \\
$\begin{array}{l}\text { Moisture content of } \\
\text { logging residues } \\
\text { Moisture content of } \\
\text { sawmill residues }\end{array}$ & 45 & $\%$ (at forest landing) \\
\hline
\end{tabular}

Table S2. Fuel and material inputs for site preparation, planting, and stand management. Natural regeneration is assumed for Northeast/North Central, thus there are no energy/material inputs.

\begin{tabular}{|c|c|c|c|c|}
\hline Inputs & $\begin{array}{l}\text { Pacific } \\
\text { Northwest }\end{array}$ & $\begin{array}{l}\text { Inland } \\
\text { Northwest }\end{array}$ & South East & $\begin{array}{c}\text { Northeast/North- } \\
\text { Central }\end{array}$ \\
\hline \multicolumn{5}{|l|}{ Energy Use (L/ha) } \\
\hline Diesel & 19.5 & 88.0 & 123.1 & \\
\hline Gasoline & 4.2 & - & - & \\
\hline Lubricants & 0.4 & 1.8 & 2.5 & \\
\hline Kerosene/Jet Fuel & 7.9 & - & - & \\
\hline Electricity & - & - & -... & \\
\hline \multicolumn{5}{|l|}{ Fertilizer Use (grams/ha) } \\
\hline Nitrogen & 71,734 & 37.0 & 103,100 & \\
\hline $\mathrm{P}_{2} \mathrm{O}_{5}$ & 0 & 62.0 & 12,800 & \\
\hline $\mathrm{K}_{2} \mathrm{O}$ & - & 152.0 & - & \\
\hline $\mathrm{CaCO}_{3}$ & - & - & - & \\
\hline \multicolumn{5}{|l|}{ Pesticide Use (grams/ha) } \\
\hline Herbicide & 4,437 & - & 1,360 & \\
\hline Insecticide & - & - & - & \\
\hline
\end{tabular}


Table S3. Energy inputs for logging in the Pacific Northwest region. Data is adapted from Oneil et al. (2017). ${ }^{1}$

\begin{tabular}{cccc}
\hline Steps & Equipment & Diesel Use (L/m3) & Lubricants $(\mathbf{L} / \mathbf{m} 3)$ \\
\hline Felling & Large feller-buncher & 0.77 & 0.01 \\
Yarding & Shovel yarder & 3.56 & 0.06 \\
Sorting & Large loader & 0.35 & 0.01 \\
Processing & Processor & 1.01 & 0.02 \\
Loading & Large loader & 0.71 & 0.01 \\
Sub-total & & $\mathbf{6 . 3 9}$ & $\mathbf{0 . 1 1}$ \\
\hline
\end{tabular}

Table S4. Energy inputs for logging in the Inland Pacific Northwest region. Data is adapted from Oneil et al. (2010). ${ }^{2}$

\begin{tabular}{cccc}
\hline Steps & Equipment & Diesel Use (L/m3) & Lubricants $(\mathbf{L} / \mathbf{m 3})$ \\
\hline Felling & Large feller-buncher & 0.37 & 0.01 \\
Skidding & Large grapple skidder & 1.13 & 0.02 \\
Processing & Processor & 0.29 & 0.01 \\
Loading & Large loader & 0.77 & 0.01 \\
Sub-total & & $\mathbf{2 . 5 5}$ & $\mathbf{0 . 0 5}$ \\
\hline
\end{tabular}

Table S5. Energy inputs for logging in the Northcentral region (except Tennesse). Data is adapted from Oneil et al. (2010). ${ }^{2}$

\begin{tabular}{cccccc}
\hline Steps & Equipment & \multicolumn{2}{c}{ Diesel Use (L/m3) } & \multicolumn{2}{c}{ Lubricants (L/m3) } \\
\cline { 3 - 6 } & & Softwood & Hardwood & Softwood & Hardwood \\
\hline Felling & Large feller-buncher & 1.02 & 0.07 & 0.02 & 0.00 \\
Skidding & Large grapple skidder & 1.66 & 2.73 & 0.03 & 0.05 \\
Processing & Processor & 0.02 & 0.02 & 0.00 & 0.00 \\
Loading & Large loader & 1.44 & 1.44 & 0.03 & 0.03 \\
Sub-total & & $\mathbf{4 . 1 4}$ & $\mathbf{4 . 2 7}$ & $\mathbf{0 . 0 7}$ & $\mathbf{0 . 0 8}$ \\
\hline
\end{tabular}

Table S6. Energy inputs for logging in Tennesse. Data is adapted from Abbas et al. (2018). ${ }^{3}$

\begin{tabular}{lcc}
\hline Type & Softwood & Hardwood \\
\hline Gasoline (L/dry ton) & 1.32 & 1.50 \\
Diesel (L/dry ton) & 9.03 & 9.62 \\
Lubricants (L/dry ton) & 0.64 & 0.68 \\
\hline
\end{tabular}


Table S7. Energy inputs for logging in the Northeast. Data is adapted from Quinn et al. (2020). ${ }^{4}$

\begin{tabular}{lcc}
\hline Steps & Diesel fuel (L/dry ton) & Lubricants (L/dry ton) \\
\hline Felling, skidding, and BMP & 6.078 & 0.122 \\
Processing pulpwood & 0.615 & 0.012 \\
Subtotal & $\mathbf{6 . 6 9 3}$ & $\mathbf{0 . 1 3 4}$ \\
\hline
\end{tabular}

Table S8. Energy inputs for logging in the Southeast region. Data is adapted from Lan et al. (in review). ${ }^{5}$

\begin{tabular}{cccc}
\hline Steps & Equipment & Diesel Use (L/m3) & Lubricant (L/m3) \\
\hline Logging & Not specified & 1.98 & 0.04 \\
Subtotal & & $\mathbf{1 . 9 8}$ & $\mathbf{0 . 0 4}$ \\
\hline
\end{tabular}

Table S9. Energy inputs for logging residues recovery. Data is adapted from Han et al. (2018) ${ }^{6}$ and Lan et al. (in review). ${ }^{5}$

\begin{tabular}{cccc}
\hline Fuel Consumption & Southeast & $\begin{array}{c}\text { Pacific Northwest } \\
\text { and Inland } \\
\text { Pacific Northwest }\end{array}$ & $\begin{array}{c}\text { Northeast/North } \\
\text { Central }\end{array}$ \\
\hline Diesel (L/dry ton) & 2.89 & 3.45 & 2.45 \\
Gasoline (L/dry ton) & - & 0.06 & - \\
Lubricants (L/dry ton) & - & 0.07 & - \\
\hline
\end{tabular}

Table S10. Energy inputs for wood pellets production. Data for Southeast and Pacific Northwest regions are derived from Morrison and Gordon $(2017)^{7}$ and Brackely et al. (2017), ${ }^{8}$ respectively.

\begin{tabular}{ccccc}
\hline & \multicolumn{2}{c}{ Southeast Region } & \multicolumn{2}{c}{ Pacific Northwest Region } \\
\cline { 2 - 4 } Energy inputs & Drying & Pelletizing & Drying & Pelletizing \\
\hline Diesel (L/dry ton) & - & 2.5 & - & - \\
Lubricants (L/dry ton) & - & 0.3 & - & 145.8 \\
Electricity (KWh/dry ton) & 40.6 & 162.2 & - & 0.2 \\
Natural Gas (L/dry ton) & - & 0.01 & 30.0 & - \\
Biomass (waste wood, MJ/dry \\
ton)
\end{tabular}


Table S11. Break-down of life cycle GHG emissions by state for the pulpwood (softwood) to electricity pathway. States with zero transportation emissions indicate that pulpwood is not included in biomass supply mix simulated by LURA; values for other stages are included here for reference.

\begin{tabular}{lrrrrrrrrrrrr}
\hline Breakdown by stages & \multicolumn{1}{c}{ WA } & \multicolumn{1}{c}{ CA } & \multicolumn{1}{c}{ ID } & \multicolumn{1}{c}{ AZ } & MN & IN & NY & ME & TN & NC & GA & LA \\
\hline Forest Management & 2.5 & 2.5 & 2.5 & 5.6 & 0.0 & 0.0 & 0.0 & 0.0 & 0.0 & 5.6 & 5.6 & 5.6 \\
Harvest/Collection & 20.6 & 20.6 & 25.1 & 15.2 & 32.2 & 24.1 & 21.7 & 23.0 & 37.3 & 15.2 & 15.2 & 15.2 \\
Biomass processing & 6.3 & 6.3 & 6.5 & 6.3 & 6.7 & 6.3 & 6.3 & 6.7 & 6.3 & 6.3 & 6.3 & 6.3 \\
Lumber mill operation & 0.0 & 0.0 & 0.0 & 0.0 & 0.0 & 0.0 & 0.0 & 0.0 & 0.0 & 0.0 & 0.0 & 0.0 \\
Transportation & 5.1 & 9.3 & 11.1 & 13.0 & 7.9 & 10.5 & 10.0 & 10.3 & 13.3 & 6.7 & 9.4 & 0.0 \\
Electricity generation & 20.0 & 20.0 & 20.0 & 20.0 & 20.0 & 20.0 & 20.0 & 20.0 & 20.0 & 20.0 & 20.0 & 20.0 \\
\hline Sum & 54.4 & 58.6 & 65.2 & 60.1 & 66.8 & 60.9 & 58.0 & 60.0 & 76.9 & 53.8 & 56.5 & 47.1 \\
\hline
\end{tabular}

Table S12. Break-down of life cycle GHG emissions by state for the logging residues (softwood) to electricity pathway. States with zero transportation emissions indicate that logging residues are not included in biomass supply mix simulated by LURA; values for other stages are included here for reference.

\begin{tabular}{lrrrrrrrrrrrr}
\hline Breakdown by stages & \multicolumn{1}{c}{ WA } & \multicolumn{1}{c}{ CA } & \multicolumn{1}{c}{ ID } & AZ & MN & IN & NY & ME & TN & NC & GA & LA \\
\hline Forest Management & 0.0 & 0.0 & 0.0 & 0.0 & 0.0 & 0.0 & 0.0 & 0.0 & 0.0 & 0.0 & 0.0 & 0.0 \\
Harvest/Collection & 11.3 & 11.3 & 11.6 & 9.4 & 8.2 & 9.4 & 9.4 & 8.2 & 9.4 & 9.4 & 9.4 & 9.4 \\
Biomass processing & 12.4 & 12.4 & 12.8 & 12.5 & 13.3 & 12.5 & 12.5 & 13.3 & 12.5 & 12.5 & 12.5 & 12.5 \\
Lumber mill operation & 0.0 & 0.0 & 0.0 & 0.0 & 0.0 & 0.0 & 0.0 & 0.0 & 0.0 & 0.0 & 0.0 & 0.0 \\
Transportation & 0.0 & 0.0 & 13.7 & 16.8 & 9.1 & 14.2 & 0.0 & 14.3 & 5.3 & 0.0 & 5.2 & 0.0 \\
Electricity generation & 20.0 & 20.0 & 20.0 & 20.0 & 20.0 & 20.0 & 20.0 & 20.0 & 20.0 & 20.0 & 20.0 & 20.0 \\
\hline Sum & 43.7 & 43.7 & 58.1 & 58.7 & 50.6 & 56.1 & 41.9 & 55.8 & 47.2 & 41.9 & 47.1 & 41.9 \\
\hline
\end{tabular}

Table S13. Break-down of life cycle GHG emissions by state for the mill residues (sawdust and shavings, softwood) to electricity pathway. States with zero transportation emissions indicate that mill residues are not included in biomass supply mix simulated by LURA; values for other stages are included here for reference.

\begin{tabular}{lrrrrrrrrrrrr}
\hline Breakdown by stages & \multicolumn{1}{c}{ WA } & \multicolumn{1}{c}{ CA } & \multicolumn{1}{c}{ ID } & \multicolumn{1}{c}{ AZ } & MN & IN & NY & ME & TN & NC & GA & LA \\
\hline Forest Management & 0.0 & 0.0 & 0.0 & 0.0 & 0.0 & 0.0 & 0.0 & 0.0 & 0.0 & 0.0 & 0.0 & 0.0 \\
Harvest/Collection & 0.0 & 0.0 & 0.0 & 0.0 & 0.0 & 0.0 & 0.0 & 0.0 & 0.0 & 0.0 & 0.0 & 0.0 \\
Biomass processing & 2.3 & 2.3 & 2.3 & 2.3 & 2.4 & 2.3 & 2.3 & 2.4 & 2.3 & 2.3 & 2.3 & 2.3 \\
Lumber mill operation & 1.5 & 1.5 & 2.7 & 2.3 & 2.5 & 2.3 & 2.3 & 2.5 & 2.3 & 2.3 & 2.3 & 2.3 \\
Transportation & 6.2 & 19.2 & 17.3 & 50.9 & 7.3 & 18.6 & 13.3 & 16.1 & 20.4 & 0.0 & 14.9 & 12.3 \\
Electricity generation & 20.0 & 20.0 & 20.0 & 20.0 & 20.0 & 20.0 & 20.0 & 20.0 & 20.0 & 20.0 & 20.0 & 20.0 \\
\hline Sum & 29.9 & 42.9 & 42.3 & 75.4 & 32.2 & 43.3 & 37.9 & 41.0 & 45.0 & 24.5 & 39.4 & 36.8 \\
\hline
\end{tabular}


Table S14. Break-down of life cycle GHG emissions by state for the wood pellet (sourced from pulpwood, softwood) to electricity pathway. States with zero transportation emissions indicate that mill residues are not included in biomass supply mix simulated by LURA; values for other stages are included here for reference.

\begin{tabular}{lrrrrrrrrrrrr}
\hline Breakdown by stages & \multicolumn{1}{c}{ WA } & \multicolumn{1}{c}{ CA } & \multicolumn{1}{c}{ ID } & AZ & MN & IN & NY & ME & TN & NC & GA & LA \\
\hline Forest Management & 0.0 & 0.0 & 0.0 & 0.0 & 0.0 & 0.0 & 0.0 & 0.0 & 0.0 & 0.0 & 0.0 & 0.0 \\
Harvest/Collection & 0.0 & 0.0 & 0.0 & 0.0 & 0.0 & 0.0 & 0.0 & 0.0 & 0.0 & 0.0 & 0.0 & 0.0 \\
Biomass processing & 89.8 & 89.8 & 92.7 & 92.5 & 95.7 & 93.3 & 95.9 & 95.7 & 95.6 & 92.5 & 92.5 & 92.5 \\
Lumber mill operation & 1.1 & 1.1 & 2.4 & 1.7 & 1.9 & 1.9 & 1.9 & 1.9 & 1.9 & 1.7 & 1.7 & 1.7 \\
Transportation & 0.0 & 0.0 & 31.0 & 33.2 & 4.3 & 7.6 & 0.0 & 4.7 & 16.3 & 10.0 & 0.0 & 0.0 \\
Electricity generation & 20.0 & 20.0 & 20.0 & 20.0 & 20.0 & 20.0 & 20.0 & 20.0 & 20.0 & 20.0 & 20.0 & 20.0 \\
\hline Sum & 110.8 & 110.8 & 146.0 & 147.4 & 122.0 & 122.8 & 117.9 & 122.4 & 133.8 & 124.2 & 114.2 & 114.2 \\
\hline
\end{tabular}

Table S15. Break-down of life cycle GHG emissions by state for the wood pellet (sourced from pulpwood) to electricity pathway. States with zero transportation emissions indicate that mill residues are not included in biomass supply mix simulated by LURA; values for other stages are included here for reference.

\begin{tabular}{lrrrrrrrrrrrr}
\hline Breakdown by stages & WA & \multicolumn{1}{c}{ CA } & ID & AZ & MN & \multicolumn{1}{c}{ IN } & NY & ME & TN & NC & GA & LA \\
\hline Forest Management & 2.5 & 2.5 & 2.5 & 5.6 & 0.0 & 5.8 & 0.0 & 0.0 & 0.0 & 5.6 & 5.6 & 5.6 \\
Harvest/Collection & 20.6 & 20.6 & 25.1 & 15.2 & 32.2 & 24.9 & 23.0 & 23.0 & 38.5 & 15.2 & 15.2 & 15.2 \\
Biomass processing & 89.8 & 89.8 & 92.7 & 92.5 & 95.7 & 93.3 & 95.9 & 95.7 & 95.6 & 92.5 & 92.5 & 92.5 \\
Lumber mill operation & 0.0 & 0.0 & 0.0 & 0.0 & 0.0 & 0.0 & 0.0 & 0.0 & 0.0 & 0.0 & 0.0 & 0.0 \\
Transportation & 0.0 & 0.0 & 31.0 & 33.2 & 4.3 & 7.6 & 0.0 & 4.7 & 16.3 & 10.0 & 0.0 & 0.0 \\
Electricity generation & 20.0 & 20.0 & 20.0 & 20.0 & 20.0 & 20.0 & 20.0 & 20.0 & 20.0 & 20.0 & 20.0 & 20.0 \\
\hline Sum & 132.8 & 132.8 & 171.2 & 166.5 & 152.2 & 151.6 & 138.9 & 143.4 & 170.4 & 143.4 & 133.4 & 133.4 \\
\hline
\end{tabular}

Table S16. Comparison of economic value versus mass based allocation factors used for the sawing process (log to rough green lumber) at lumber mill. States within the same region share the same allocation factors.

\begin{tabular}{|c|c|c|c|c|c|c|c|c|c|c|}
\hline \multirow[b]{2}{*}{ Products (green) } & \multicolumn{5}{|c|}{ Mass-based allocation } & \multicolumn{5}{|c|}{ Economic Allocation } \\
\hline & PNW & INW & $\mathrm{SE}$ & $\begin{array}{c}\mathrm{NE} / \mathrm{NC} \\
(\mathrm{SW})\end{array}$ & $\begin{array}{c}\mathrm{NE} / \mathrm{NC} \\
(\mathrm{HW})\end{array}$ & PNW & INW & SE & $\begin{array}{c}\mathrm{NE} / \mathrm{NC} \\
(\mathrm{SW})\end{array}$ & $\begin{array}{c}\mathrm{NE} / \mathrm{NC} \\
(\mathrm{HW})\end{array}$ \\
\hline lumber & $50.1 \%$ & $52.0 \%$ & $52.2 \%$ & $34.0 \%$ & $59.4 \%$ & $91.8 \%$ & $92.0 \%$ & $91.4 \%$ & $85.1 \%$ & $95.1 \%$ \\
\hline chips & $24.4 \%$ & $32.0 \%$ & $30.7 \%$ & $37.0 \%$ & $15.5 \%$ & $5.7 \%$ & $7.2 \%$ & $6.9 \%$ & $11.8 \%$ & $3.2 \%$ \\
\hline Sawdust/shavings & $5.6 \%$ & $4.0 \%$ & $9.7 \%$ & $11.0 \%$ & $13.6 \%$ & $0.7 \%$ & $0.5 \%$ & $1.1 \%$ & $1.8 \%$ & $1.4 \%$ \\
\hline hogfuel & $13.8 \%$ & $1.0 \%$ & $4.8 \%$ & $5.0 \%$ & $0.2 \%$ & $1.7 \%$ & $0.1 \%$ & $0.6 \%$ & $0.8 \%$ & $0.0 \%$ \\
\hline bark & $6.1 \%$ & $9.0 \%$ & $2.6 \%$ & $13.0 \%$ & $11.2 \%$ & $0.2 \%$ & $0.2 \%$ & $0.1 \%$ & $0.5 \%$ & $0.3 \%$ \\
\hline
\end{tabular}


Table S17. Comparison of economic value versus mass based allocation factors used for the planning process (rough saw lumber to planned lumber) at lumber mill. States within the same region share the same allocation factors.

\begin{tabular}{lrrrrrrrrrr}
\hline & \multicolumn{4}{c}{ Mass-based allocation } & \multicolumn{4}{c}{ Economic Allocation } \\
\cline { 2 - 12 } Products & \multicolumn{1}{c}{ PNW } & \multicolumn{1}{c}{ INW } & \multicolumn{1}{c}{ SE } & $\begin{array}{c}\text { NE/NC } \\
\text { (SW) }\end{array}$ & $\begin{array}{r}\text { NE/NC } \\
\text { (HW) }\end{array}$ & PNW & INW & SE & $\begin{array}{c}\text { NE/NC } \\
\text { (SW) }\end{array}$ & $\begin{array}{c}\text { NE/NC } \\
(\mathrm{HW})\end{array}$ \\
\hline Sawn lumber & $88.7 \%$ & $82.0 \%$ & $90.1 \%$ & $90.0 \%$ & $80.2 \%$ & $99.1 \%$ & $98.6 \%$ & $99.3 \%$ & $99.2 \%$ & $98.4 \%$ \\
Chips & $1.3 \%$ & $0.0 \%$ & $0.2 \%$ & $1.0 \%$ & $0.0 \%$ & $0.2 \%$ & $0.0 \%$ & $0.0 \%$ & $0.1 \%$ & $0.0 \%$ \\
Sawdust/shavings & $10.1 \%$ & $18.0 \%$ & $9.6 \%$ & $9.0 \%$ & $19.8 \%$ & $0.7 \%$ & $1.4 \%$ & $0.7 \%$ & $0.7 \%$ & $1.6 \%$ \\
\hline
\end{tabular}

